Aus dem kommunalen Krankenhause Västerbottens in UmeâSchweden (dirigierender Arzt: Dr. med. K.H.Giertz).

\title{
Eine neue Modifikation der Sauerbruchschen Unterdruckkammer.
}

\author{
Von K. H. Giertz. \\ (Mit 5 Abbildungen.)
}

Seitdem Sauerbruch im Jahre Igo4 seine geniale Wethode gegen die operative Pneumothoraxgefahr angegeben hat, ist von verschiedenen Seiten eine rastlose Arbeit auf die Vereinfachung der Apparate verwendet worden. Eine große Anzahl von Apparaten -- meistens vom Überdrucktypus - sind im Markte. Der praktisch tätige Chirurg bedarf so einfacher, handlicher und billiger Apparate wie möglich. Es ist aus diesen Gesichtspunkten natürlich, daß die ersten Lnterdruckkammern durch die Überdruckapparate mit Kopfkasten verdrängt wurden, dal.3 diese ihrerseits den $\ddot{U}$ berdruckapparaten mit Maske weichen mußten, und daß schließlich die Insufflation von Meltzer-Auer auch diese ein Uberdruckverfahren mit noch einfacherer Apparatur ...- von vielen als das Instrumentarium der Zukunft bei Operationen an dem geöffneten Brustkasten angesehen wird.

Die wichtigsten Einwendungen gegen dic zurzeit angewandten Unterdruckkammern (Sauerbruchs und Willy Meyers) sind folgende. Die Anschaffungskosten sind zu hoch, ihre Anwendung ist umständlich. Dazu kommt -- vielleicht der Hauptgrund, daß der jetzige Unterdruckkammertypus nicht durchgedrungen ist , daß der Operateur und Narkotiseur durch eine Wand geschieden werden, und daß der Patient an die Wand festgelegt wird, was trotz beweglichem Halsring und Ausbau der Kopfwand zur Folge hat, daß die Möglichkeit einer Veränderung der Lage des Patienten während der Operation beschränkt und die ,Fllenbogenfreiheit" des Operateurs und der Assistenten vermindert wird. 
Viele Überdruckapparate mit Maske - z. B. Tiegels, Lotschs, Schoemakers und Steinmanns - sind ja sehr einfach und betriebssicher. Die Erfahrung lehrt, daß sie, wenn niedrige Druckwerte angewandt werden, auch ungefährlich sind.

Meiner Ansicht nach ist es bei den Überdruckapparaten ein Vorteil, daß man nicht allein an Sauerstoff gebunden ist. Der Sauerstoff ist im Betrieb nicht so billig. Er ist wenigstens an entlegenen Orten teuer und beschwerlich anzuschaffen. Schließlich - worüber auch viele Chirurgen klagen - der Sauerstoffzylinder wird vielmals gerade in dem Augenblicke leer, wenn der Überdruckapparat am meisten vonnöten ist.

Die Insufflationsapparate sind gewiß einfacher und billiger als die gewöhnlichen Überdruckapparate mit Maske. Mir scheint jedoch das Insufflationsverfahren so viel schwieriger und gefährlicher als das Überdruckverfahren mit einem gewöhnlichen Maskenapparat, daß es mir zweifelhaft ist, ob mit dem Umtausch des Maskenapparats gegen einen Insufflationsapparat etwas gewonnen ist. Wenigstens ist eine Warnung gegen die Insufflation am Platze. Die Nachteile, die der Insufflation zur Last gelegt werden können, sind nicht wenige.

Selbst die Einführung des Katheters ist schon ein Eingriff, dessen Gefahr zwar in der Hand eines geübten Operateurs nicht $z u$ hoch angeschlagen zu werden braucht, der aber doch bei einem Maskenapparat nicht in Frage kommt. Eine gewisse Verantwortlichkeit ist auch damit verbunden, daß man aufpassen muß, daß der Katheter nicht zu tief hineindringt, da sonst direkte Lebensgefahr entstehen kann.

Die Voraussetzung der Einführung des Katheters ist eine tiefe Narkose. Eine Operation in Lokalanästhesie kann bei der Insufflationsmethode nicht in Frage kommen. Bei Operationen von Lungenabszessen bei freier Pleura sowie bei großen Thorakoplastiken in Lokalanästhesie habe ich meine Druckdifferenzkammer, die anwendbar ist, trotzdem der Patient wach ist oder nur einige Tropfen Narkosemittel bekommen hat, schätzen gelernt.

Bei Glottiskrampf beim Insufflationsverfahren entsteht ein Hindernis für die Ausblasung der Luft sowie für die Expektoration, in beiden Fällen mit bedrohlichen Symptomen. Das Um- 
Eine neue Modifikation der Sauerbruchschen Unterdruckkammer. $\quad$ I69

tauschen des Katheters, wenn dies während der Operation geschehen muß, kann wenigstens, wenn keine sachkundige Hilfe zur Hand ist, so viel Zeit in Anspruch nehmen, daß wirkliche Gefahr entstehen kann.

Diese letzterwähnten Gefahren können vermieden werden, wenn die Insufflation nach der $\mathrm{Kuhnschen} \mathrm{Methode} \mathrm{gemacht}$ wird. Zuerst wird die perorale Intubation mit dem Kuhnschen Rohre gemacht, in welchem dann der Katheter bis zur gewünschten Tiefe eingeführt wird.

Schließlich - und dies ist sowohl beim Tierexperiment als bei Narkosen an Menschen vorgekommen - kann ein Mediastinalemphysem entweder durch eine Verwundung der Schleimhaut durch den Katheter oder durch eine Lungenruptur entstehen.

In einem Falle (L uke) ist der Katheter in den rechten Bronchus geglitten. Das Mißgeschick wurde vom Narkotiseur nicht rechtzeitig bemerkt. Die Einblasung wurde mit der Folge fortgesetzt, daß Pat. an Lungenruptur starb.

Man sollte meinen, daß die Frage nach der Überlegenheit des Unter- oder des Überdruckverfahrens durch die praktische Entwicklung als erledigt gelten könnte. Das ist jedoch nicht der Fall. Gerade in letzter Zeit ist diese Streitfrage von verschiedenen Seiten zur Diskussion aufgenommen worden mit dem Resultat, daß die Beobachtungen Sa u erbruchs vom Jahre I 904 sich bestätigen.

In kurzen Zügen ist die geschichtliche Entwicklung dieser Frage die folgende. Schon zu Anfang seiner Versuche, das Pneumothoraxproblem zu lösen, hatte $\mathrm{Sauerbruch}$ beim Tierexperiment zahlreiche Störungen der Zirkulation beim Überdruck beobachtet, was ihn veranlaßte, bei der Konstruktion einer Kammer für Menschen ganz und gar vom Überdruck abzusehen.

B rauer (I905) hielt die beiden Verfahren für völlig gleichwertig und meinte, das einzig Ausschlaggebende sei die Druckdifferenz, ohne Rücksicht darauf, wie diese zustande kommt.

Tiegel (1905) machte geltend, der Unterdruck sei physiologisch, der Überdruck dagegen nicht, und er warnte direkt vor der Anwendung von Überdruck bei Ösophagusoperationen, bei kranken Lungen und Herzen sowie bei Mediastinalkrankheiten. Bei seinen damaligen Untersuchungen hatte Tiegel mit so hohen Druckdifferenzwerten gearbeitet, wie sie in der Wirklichkeit kaum zur Anwendung kommen können. 
Seidel (1906) studierte Atmungs- und Kreislaufsverhältnisse an Hunden und Kaninchen. Er kam zu dem Resultat, daß die Veränderungen, die durch das Überdruckverfahren bedingt sind bei richtiger Anwendung, das heißt, wenn man möglichst geringen Überdruck und nur so viel anwendet, daß man dic Ineumothoraxgefahr ausschaltet, so gering sind, daß sie keine Rolle spielen. Seidel führte keine parallelen Untersuchungen mit Unterdruck aus.

Dreyer und Spann hus (1908) haben dagegen umfassende Paralleluntersuchungen zwischen Überdruck ( $\mathrm{Br}$ a u e r $\mathrm{s} \Lambda$ pparat) und Interdruck (S a u e r b r c chs Kammer) angestellt. Sic stellten fest, daß der Unterdruck betreffs der Druckverhältnisse der Kreisläufe auch nicht ohne Einwirkung war. Beim Unterdruck stellten sie eine Steigerung des Druckes im kleinen, ein Sinken im großen Kreislaufe, beim Uberdruck ebenso eine Steigerung im kleinen, cin Sinken im großen fest. Sie fanden weiter beim Über-wie auch beim Unterdruck eine Steigerung des Venendruckes. Sie zeichneten die Luftschwankungen in der Trachea bei Über- und bei Unterdruck auf. Die Kurven verliefen übereinstimmend. Auch die Bewegungen des Brustkastens waren bei beiden Verfahren gleich.

Da gleichzeitig dic praktische Erfahrung mit Überdruckapparaten in günstiger Richtung verliefen, gewann die Ansicht allgemeine Anerkennung, daß Über- und Unterdruck völlig gleichwertig seien. (Küttner, Schmieden, Meyer, Robinson, Körte.)

$\mathrm{S}$ a u e rbruch hält dagegen die beiden Verfahren andauernd für nicht glcichwertig, gibt aber zu (1910), daß es den Unterschied übertrieben hat und $\mathrm{daB}$ in der Praxis beide Methoden ohne Gefahr angewandt werden können.

Tiegel hat durch den Bau seines Überdruckapparates (1908) und durch die Anwendung desselben bei Menschen gezeigt, daß er den praktischen Unterschied bedeutungslos fand. Theoretisch hält er dagegen an seiner alten Ansicht fest. In einer experimentellen Arbeit voll I9I I hat er die Resultate einer Paralleluntersuchung über die verschiedenen Einwirkungen der beiden Verfahren auf Blutdruck und Herz mitgeteili. Ti e g el konnte bei diesen Versuchen unmittelbar nacheinander Unter- oder Überdruck etablieren. I) as Tier liegt nämlich mit luftdicht schlieBender IIalsmanschette, den Körper in einer größeren, den Kopf in einer kleineren Kammer. In der größeren kann Lnterdruck, in der kleineren Überdruck hergestellt werden. Tiegel stellt auf diese Weise eine Steigerung des Drucks sowohl in den großen Thorakalvenen wie in der Lungenarterie beim $t$ berdruck fest. Ietzteres weist auf einen erhöhten Widerstand der Blutzirkulation im kleinen Kreislaufe hin und bringt eine gesteigerte Arbeit für die rechten Herzkammern, die zur Dehnung derselben führt, mit sich. Beim Übergang zum Unterdruck verschwinden alle diese Symptome. Beim Unterdruck wird der Druck in den groBen Thorakal- 
venen physiologisch, bei größeren Druckdifferenzen (IO-I $5 \mathrm{~cm}$ Wasser) sogar etwas vermindert. Der Druck in der Lungenarterie steigt beim Überdruck über den bei Lungenkollaps bestehenden, beim Unterdruck sinkt er. Die Durchblutung der Lungen ist also nach Tiegel beim Überdruck erschwert, beim Unterdruck erleichtert. Der Druck in den peripheren Arterien wird beim Linterdruck etwas gesenki, was wahrscheinlich davon herrührt, daB der Gefäßwiderstand durch die Erweiterung der peripheren Kapillargebiete vermindert wird.

In ciner neuen Arbeit von $\mathrm{IgI} 2$ hat $T$ iegel sich ron neuem eingehend mit diesen Fragen beschäftigt. Er zeigt auf eine klare und beweisende Art die Verschiedenheit der physikalischen Phänomene bei Lber- und Unterdruck. Tiegel macht darauf aufmerksam, wie eine große Anzahl von ganz elementaren physikalischen Begriffen in der Diskussion auf den Kopf gestellt wurden.

Zu bedenken ist, daß, obwohl der Effeht bei beiden Verfahren derselbe ist, nämlich die Entwicklung der Lunge, er doch in dem einen Falle durch eine Aufblasung von innen, in dem anderen Falle durch eine Saugung an der Lungenoberfläche von außen gewonnen wird.

Tiegels Resultate stehen in mehreren Hinsichten in krassem Widerspruch zu Dreycrs und Spannhaus', der seine Erklä. rung durch die verschiedene Versuchsanordnung (Aufstellung des Manometers innerhalb [Dreyer] bzw. auBerhalb [Tiegel] der Unterdruckkammer) crhält.

T i c gel zeichnete bej Unterdruck den Blutdruck in der Vena cava superior gleichzeitig mit einem Manometer in der atmosphärischen Luft und einem anderen in der Unterdruckkammer auf. Die beiden Manometer zeigten verschiedene Werte: das in der atrnosphärischen I.uft eine geringe Druckverminderung, das in der Kammer eine Erhöhung.

Da Sauerbruch und Anschütz bei ihren Versuchen über mit Unterdruck kunstlich hervorgerufene Gehirnanämie festgestellt haben, daß eine Druckerniedrigung noch weiter peripher in der Vena jugularis (auBerhalb der Kammer mit dem Manometer in der atmosphärischen Luft) vorhanden ist, so müssen die 'I i egelschen Resultate dic richtigen scin.

Cloetta hat in mehreren Arbeiten der letzteren Jahre (1910 bis 1913) diese und andere für die Lungenchirurgie und Lungentherapie wichtigen Fragen zu beleuchten versucht.

Fr hat sich in erster Reihe die alte, noch nicht klar gestellte. Frage, in welchem Zustand - Inspiration oder Exspiration - die Lunge an besten durchblutet ist, zur Beantwortung aufgestellt. Er hat dabei den von ihm konstruierten Lungenplethysmographen angewandt. Die Brustkastenwand wird resezicrt. I)ie Lunge wird in 
den gläsernen Plethysmographen bei gänzlichem Ausfall der Atembewegung ( $\mathrm{C}$ u ra r e) luftdicht eingeführt. Die Lungen können nun in jeden beliebigen Blähungszustand übergeführt und darin erhalten werden, einerseits durch $\mathrm{O}_{2}$-Einblasung von der Trachea aus (Überdruck) oder durch Luftverdünnung in dem gläsernen Pleuraraum (Unterdruck).

Die einzigen Volumveränderungen, die das durch Curare respiratorisch ruhiggestellte Organ zeigt, sind pulsatorischer Natur. Sie können sowohl direkt beobachtet, als auch graphisch quantitativ registriert werden. Wenn man indessen sicher die Größe der Blutzufuhr zu einem Organ messen will, hat man am besten die venöse Ausflußmenge zu bestimmen.

An lebenden Organen läßt sich dies nicht direkt, wohl aber indirekt machen. Die ganze Blutmenge, welche die Lungen durchströmt, geht durch den linken Vorhof und die linke Kammer in die Aorta.

Ist die Herzkraft und der Widerstand in den peripheren Gefäßen konstant, so ist der Aortendruck nur noch abhängig von der Masse des zufließenden Blutes. Auf diese Weise gibt der Aortendruck ein indirektes $\mathrm{MaB}$ für die Menge des Lungenvenenblutes ab. Mit diesen Methoden stellt C loe t t a fest, daß der Druck der Aorta bei Ausdehnung der Lunge immer sinkt, beim Lungenkollaps steigt.

Wie dieses Hindernis der Zirkulation auf der Höhe der Inspiration zustande kommt, hat $\mathrm{Cl}$ o et t a sehr hübsch durch mikroskopische Präparate demonstriert. Bei der Inspiration sind Gefäße und Kapillaren gestreckt und verengt, bei der Exspiration sind sie weit und geschlängelt.

Die weiteren Untersuchungen $C l$ lo t t a s haben bewiesen, daB die beste Durchblutung der Lungen zustande kommt, wenn die Lunge vom Kollapszustand aus minimale Bewegungen unter einem Minusdruck von $2-3 \mathrm{~mm} \mathrm{Hg}$ im Pleuraraum macht. Dieser Druck ist derselbe, den Fried rich und andere Chirurgen als genügend zur Vermeidung der Pneumothoraxgefahr nachgewiesen haben.

Von besonderem Interesse sind die Cloet t a schen Beobachtungen für das Druckdifferenzverfahren.

Mehrere Autoren sind, wie eben erwähnt, von dem falschen Standpunkt ausgegangen, daß das einzig Bedeutsame beim Druckdifferenzverfahren die vorhandene Differenz zwischen dem Druck an der äußeren und inneren Lungenfläche ist, gleichgültig, auf welche Weise diese Differenz zustande gebracht worden ist.

Wenn es sich um die Blähung eines einfachen elastischen Sackes ohne Zwischenwände und Ausbuchtungen und vor allem ohne morphologische Verschiedenheiten im Bau der Wandungen handelte, so wäre nach $v$. R o h d e n wohl theoretisch gegen die Vorstellung nichts einzuwenden, daß derselbe Grad der Lungenblähung und die gleichen 
Durchblutungsbedingungen durch einen positiven Innendruck wie durch einen gleich großen negativen Außendruck erzielt werden könnten. Nun darf aber das Lungengewebe mit dem weitverzweigten Gefäß- und Bronchialbaum in seiner Abhängigkeit von mechanischen Bedingungen keineswegs mit einem elastischen Sack verglichen werden, schon deshalb nicht, weil seine Wandungen nicht aus einer homogen zusammengesetzten elastischen Einheit bestehen, sondern ein sehr kompliziertes doppeltes Röhrensystem in sich aufnehmen müssen.

Die Versuche Cloet tas haben folgendes bewiesen:

I. Wird die Lunge nacheinander das eine Mal durch Uber-, das andere Mal durch Unterdruck bis genau zu der gleichen Vergrößerung ihres Volumens gebläht, so ist hierzu regelmäßig bei Unterdruck ca. ${ }^{1 / 3}$ weniger Kraft als bei Überdruck erforderlich; wendet man also gleich starke Druckdifferenzen bei beiden Verfahren an, so wird die Lunge beim Unterdruck entsprechend stärker gebläht.

2. Bei derselben Volumenvergrößerung der Lunge sinkt, wenn dieselbe durch Überdruck erzeugt wurde, der Aortendruck tiefer als beim entsprechenden Unterdruck.

3. Der Druck in der Arteria pulmonalis steigt bei gleicher Lungenblähung beträchtlich höher bei Anwendung von Überdruck. In Übereinstimmung mit letzterem steigt der diastolische Druck im rechten Ventrikel beim Überdruck an, beim Unterdruck nicht.

Das Resultat der Untersuchungen Cloettas ist also, daB Über- und Unterdruck nicht gleichwertig sind. Die Zirkulation leidet beim Überdruck bedeutend mehr. Diese nach Cloetta feststehenden Fakta werden folgendermaßen erklärt.

Bein Unterdruck werden die atmosphärischen Verhältnisse in der Lunge nicht verändert, es entsteht aber andererseits eine Druckerriedrigung an der Lungenoberfläche. Die Pleura pulmonalis, die allein unter diesem negativen Druck zu stehen kommt, wird nach außen gesaugt. Unter der Lungenoberfläche liegt die ganze Masse von Lungengewebe, mit der Pleura zusammengewachsen.

Dieses Gewebe wird, sofern die angewandte Kraft, d. h. der negative Druck es vermag, mitgezogen und die Alveolenräume werden mit atmosphärischer Luft gefüllt. Die ganze Entwicklung der Lunge wird durch eine völlig mechanische Saugung von außen her bewirkt. Beim Überdruck liegt die Sache ganz anders. Durch einen erhöhten Druck durch die Trachea wird die Lunge gegen die atmosphärische Luft aufgeblasen. Eine Übereinstimmung mit Unterdruck würde entstehen, wenn man sich denken könnte, daß der Überdruck seine Wirkung immer 'ganz peripher unter der Pleura pulmonalis ausuibte und die übrige Lunge frei ließe.

Die Erklärung, weshalb die verschiedenen Druckverfahren bei 
derselben Kraft nicht dieselbe Ausdehnung bewirken, liegt auf der Iland. Durch die Friktion entsteht beim Überdruck ein Kraftverlust.

Die Gefäße der I.unge müssen auch beim Überdruck verengt werden. Die geringere Durchfließungsmenge des Aortenblutes (das Sinkenl des Aortendrucks) und der größere Widerstand der Lungengefäße (das Ansteigen des Pulmonalisdrucks) sowie der erhöhte diastolische Druck des rechten Ventrikels sind objektive Anzeichen davon.

Sauerbruch hat (I9Io) mit Schenk, Lohmann und von Spee eine theoretische Erklärung dahin gegeben, daß vom anatomischen Gesichtspunkt aus beim Uberdruck eine Kompression der Lungenkapillaren unvermeidlich ist, beim Unterdruck dagegen cher eine Erweiterung mit Erleichterung der Durchblutung entsteht.

An der Diskussion hat schließlich von Rohden (Mor a w it $z$, Klinik 1913) teilgenommen. von Rohden macht auf die Gefahr von Irrtümern aufmerksam, die immer besteht, wenn nicht alle Experimente mit einer Methode, die eine Untersuchung der lebenden Lunge bei jedem gewünschten Aufblasungszustand bei normaler Zirkulation zuläßt, ausgeführt werden. von Rohden hat gasanalytische Untersuchungen mittels der $\mathrm{H}$ e B schen Methode (I9I3) angestellt, deren Prinzip darin besteht, daß nach den Veränderungen des Sauerstoffgchalts des Carotisblutes die Größe der Durchblutung der Lunge geschätzt werden kann. von Rohden hat hierbei die C loct ta schen Erfahrungen völlig bestätigt. Nach von Rohden ist es experimentell bewiesen, daß bei lebenden Organen $S$ a uerbruchs Unterdruckverfahren dem $\mathrm{Br}$ a u erschen Überdruckver. fahren überlegen ist.

Wie ersichtlich, sind die Forscher, welche sich mit dieser Frage beschäftigt haben, bei weitem nicht einig, was zu nicht geringem Teil an der Subtilität der Experimente, die zur Aufklärung der Frage notwendig sind, liegt. Die Fehlerquellen, denen man dabei ausgesetzt ist, sind groß und schwer zu überwinden.

Bei einer kritischen Durchmusterung alles dessen, was sich bei der Diskussion über die physiologische Bedeutung des Unterund, Überdrucks ergeben hat, kann als sicher festgestellt angesehen werden, daß keines der Verfahren den Organismus unbeeinflußt läßt.

Als sicher muß auch angesehen werden, daß der Unterdruck den physiologischen Verhältnissen am nächsten kommt. Die Störungen beim Überdruck sind bedeutend größer und finden ihre Erklärung durch die Tatsache, daß eine Ausdehnung der Lunge mit Überdruck ohne Kompression der Lungenkapillaren 
nicht zustande kommen kann. Diese Kompression bringt eine Drucksteigerung der Arteria pulmonalis und eine erhöhte Arbeit der rechten Herzkammer mit sich. Dies geschieht, ob Maskenapparate oder Insufflationsapparate angewandt werden.

Die Erfahrung hat gezeigt, daß geringe Grade von Utberdruck nicht gefährlich sind. Bei größeren oder langdauernden Druckdifferenzen kann wahrscheinlich die gefährliche Konsequenz der Zirkulationsstörungen hervortreten. Ob dies geschieht oder nicht, hängt von der Reseryekraft der rechten Kammer ab. Diese ist oftmals vielleicht nicht so groß. Eine Methode, diese im voraus zu beurteilen, steht uns noch nicht zur Verfügung. Bei langdauernden Operationen ist aus diesen Gründen der Unterdruck vorzuziehen.

In gewissen Fällen bietet, wie $\mathrm{S}$ a uerbruch gezeigt hat, das Unterdruckverfahren praktische Vorteile vor dem Überdruckverfahren. Dies ist bei Behandlung von Spannungspneumothorax und Mediastinalemphysem der Fall. In zwei Fällen von Mediastinalemphysem wies Sauerbruch nach, daß bei Behandlung der Patienten mit Thorakotomie in der Unterdruckskammer, der negative Druck der Kammen eine direkte Saugwirkung auf die in den Gewebemaschenräumen unter gewöhnlichem Luftdruck stehende Luft ausübte. Das gefährliche Mediastinalemphysem verschwand auf einmal, desgleichen auch das Hautemphysem. Der Überdruck steht in solchen Fällen machtlos da. Noch einen weiteren Vorteil des Unterdruckverfahrens hat Sauerbruch hervorgehoben.

Alle Tierexperimente haben gezeigt, daß die Totalexstirpation einer Lunge im Überdruck - das gilt sowohl von Operationen mit Überdruckapparaten (Robinson, Sauerbruch) wie auch von solchen mit Insufflation (Schlesinger) - - ungünstiger und mit höherer Mortalität als im Unterdruck verlaufen. Die Ursache ist die, daß beim Überdruckverfahren eine Drucksteigerung in der leeren Pleurahöhle zurückbleibt, die ein direktes Hindernis für die Verschiebung der Brustwand, des Zwerchfells, des Mediastinums und der gesunden Lunge bildet, wie sie für die Heilung notwendig ist. Aus den experimentellen Untersuchungen Friedrichs wissen wir nämlich, daß nach einer Lungenexstirpation eine völlige Anpassung des Organismus an die neuen Verhältnisse 
dadurch eintritt, daß der nach der Lungenexstirpation gebildete leere Raum durch ein Aneinanderlegen der Wände ausgeglichen wird. Dieser Prozeß ist es, dem der nach der Lungenexstirpation in Überdruck zurückbleibende erhöhte Luftdruck, der die Transudation in die Höhle hinein mit der Gefahr einer postoperativen Infektion erleichtert, entgegenwirkt.

Aus praktischen Gründen ist deswegen das Unterdruckverfahren dem Überdruckverfahren weit vorzuziehen.

Es erschien mir daher von großem Vorteil, wenn man einen Apparat konstruieren könnte, wo das Unterdruckprinzip beibehalten war, alle Vorteile des Überdruckverfahrens aber zur Geltung kamen. Als Hauptpunkte dabei müssen hervorgehoben werden: Der Apparat muß leicht hantierlich, leicht in Betrieb zu setzen wie auch betriebssicher, billig anzuschaffen und betriebsbillig sein.

Um dies zustande zu bringen habe ich in meiner aseptischen Operationsabteilung ein Zimmer als Unterdruckkammer einrichten lassen. Beim Neubau eines Krankenhauses ist es eine einfache Sache, in der Nähe des Operationssaals ein luftdichtes Zimmer behufs eventueller Einrichtung einer Unterdruckkammer zu bauen. Fin solches Zimmer wird am besten ohne Fenster, um schwer zu beseitigende Leckagen zu vermeiden, gebaut. Dieses Zimmer kann ja, wenn Raum und Ökonomie nicht besondere Säle zulassen, als Dunkelzimmer bei endoskopischen Operationen und Untersuchungen angewandt werden. Die Erfahrung lehrte mich, daß die Lecke cines solchen Zimmers nicht so leicht zu beherrschen sind, wenn es nicht mit Umsicht gebaut wird. Mehr als eine Tür sollte nicht vorhanden sein. Um die Tür ganz dicht zu bekommen, ist es notwendig, einen eisernen Rahmen fest mit Zement einzumauern, der am passendsten von $\mathrm{T}$-förmigen Winkeleisen hergestellt wird, dessen eine Hälfte in die Wand eingemauert wird, die andere als Rahmen für die 'Tür dient.

Die Tür wird aus Billigkeitsrücksichten aus gewöhnlichem Holz hergestellt und durch Gummipackung (Drainröhre) längs den Rändern gegen den Rahmen gedichtet. Um die Packung zu schonen, wenn die 'Tür, während der Nichtfunktion der Kammer, zugemacht werden muß, und um die Dichtheit zu sichern, da eine hölzerne Tür trotz trockenem Holz und bester Arbeit 
schief werden kann, kann man auf folgende Weise (wie wir auch hier in Umeå getan) verfahren.

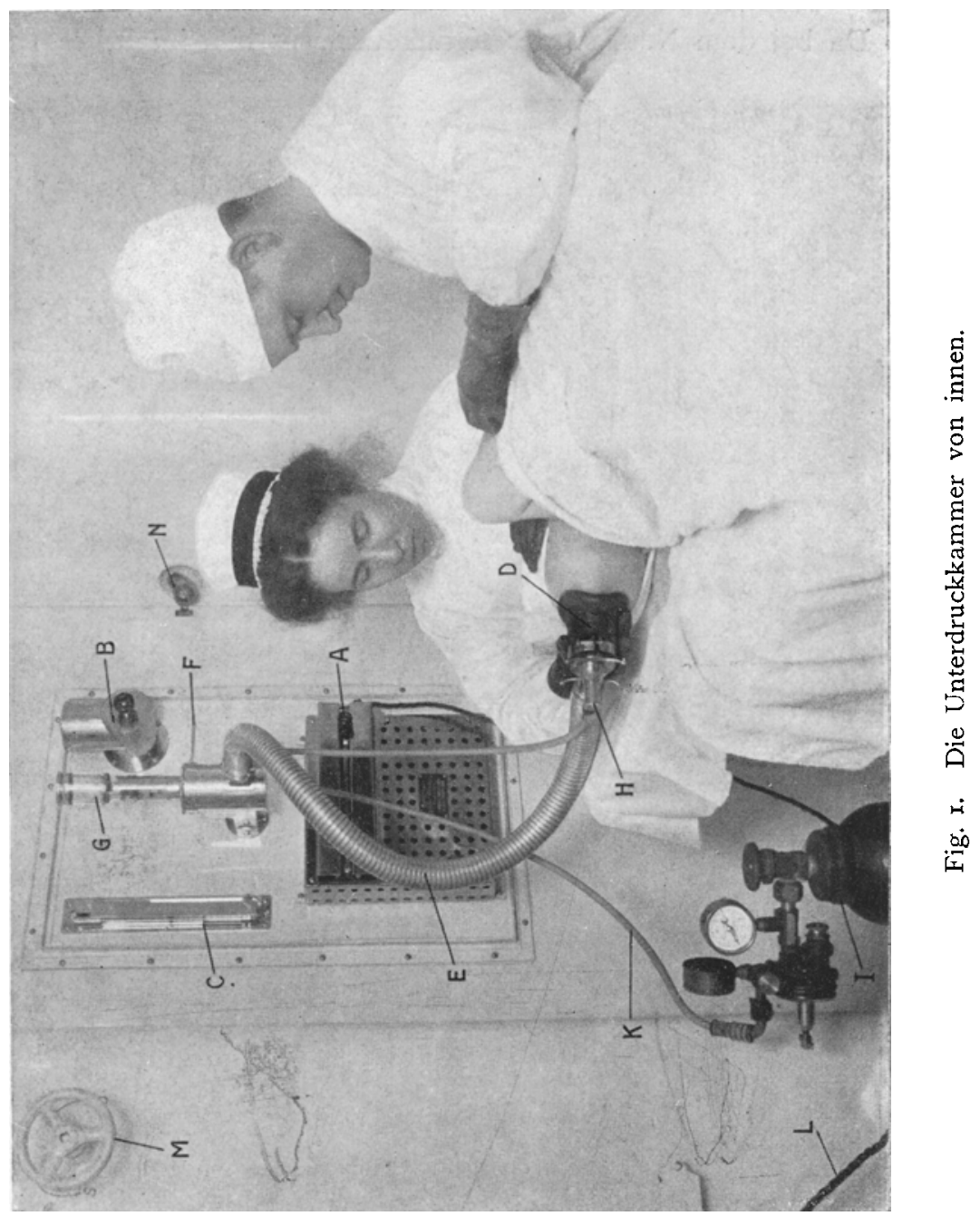

Wir haben eine Vorrichtung angebracht, die ein festes Anziehen der Tür an den Rahmen ermöglicht. Die Anordnung besteht teils aus einer durch die Wand gehenden Schraubvorrichtung (Fig. I, M), die hebelartig (Fig. 3) die Tür an den Rahmen 
zieht, teils aus zwei gewöhnlichen Schrauben (Fig. I u. 2, N), welche demselben Zwecke dienen. Die Tür hat die Größe $90 \times 208 \mathrm{~cm}$.

Da bei dem Neubau des Krankenhauses hier im Jahre I907

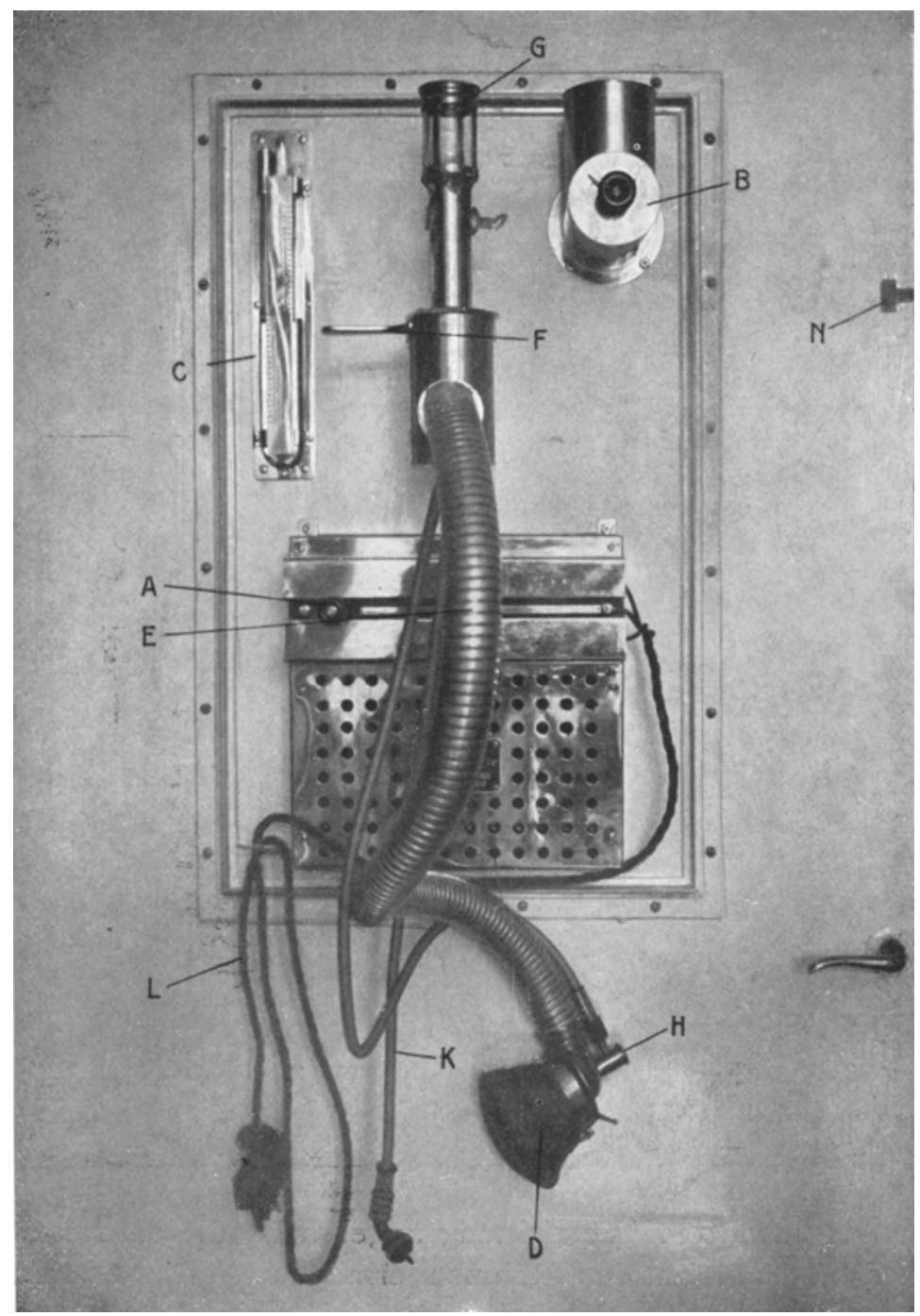

Fig. 2. Die Tür mit der Platte. 
Eine neuc Modifikation der Sauerbruchschen Unterdruckkammer. I 79 niemand daran gedacht hatte, eine Unterdruckkammer einzurichten, mußte eine solche eingerichtet werden.

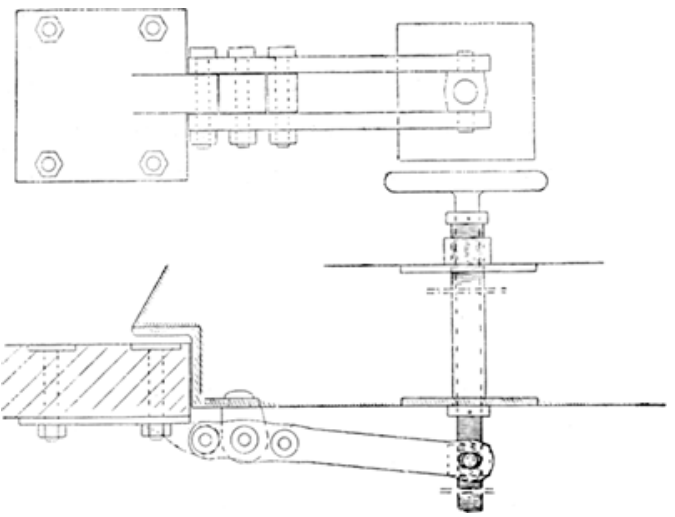

Fig. 3. Schraubvorrichtung für die Dichtung der Tür.

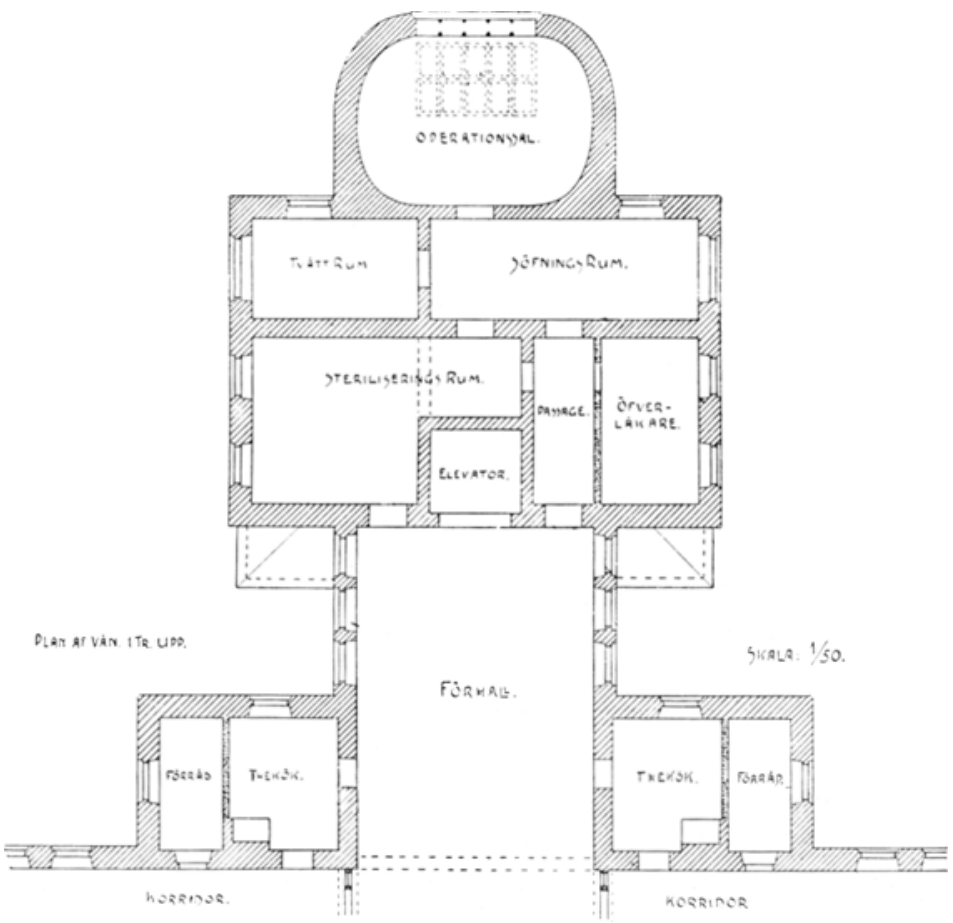

Fig. 4. Die aseptische Operationsabteilung vor der Einrichtung der Unterdruckkammer. 
Wie die Fig. 4 und 5 zeigen, umfaßt die aseptische Operationsabteilung in meinem Krankenhause einen Operationssaal mit einem danebenliegenden Wasch- und Narkosezimmer für Patienten. Ein angrenzendes Zimmer war Waschraum für Ärzte und Pflegerinnen. Außerhalb dieser Zimmer befanden sich ein kleines Instrumentenzimmer sowie ein großes kombiniertes Verband- und Sterilisierungszimmer. Von diesem letzteren Zimmer

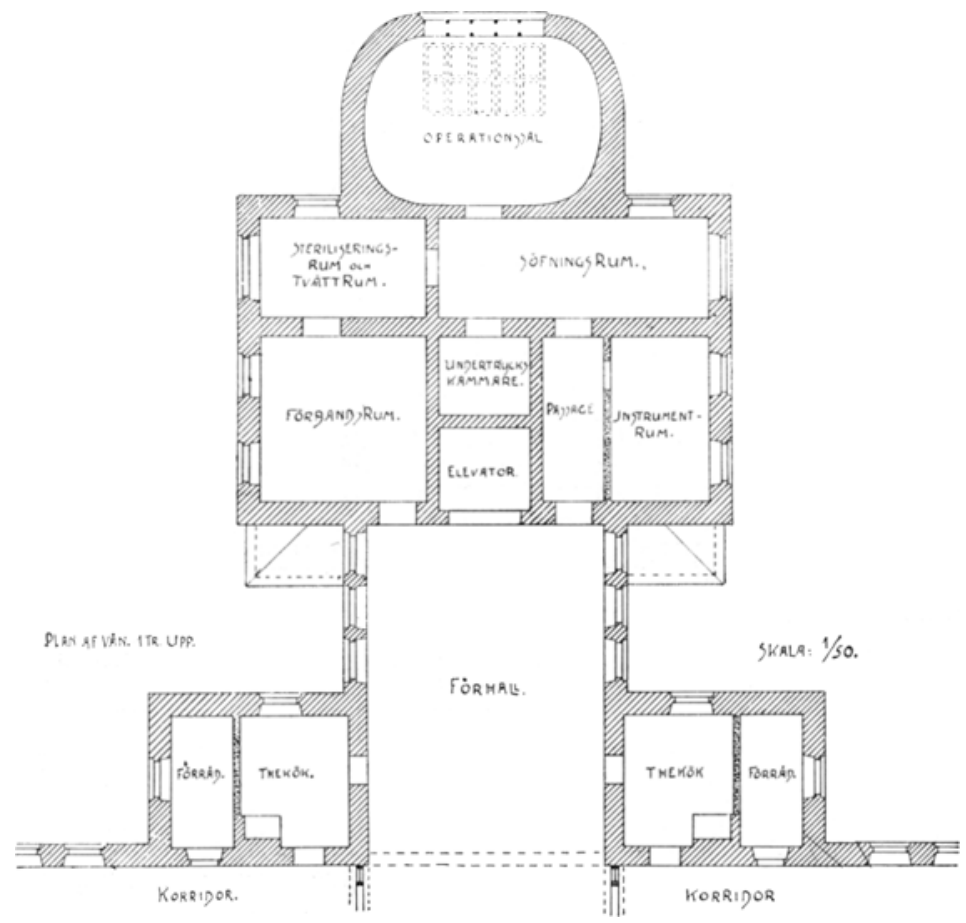

Fig. 5. Die aseptische Operationsabteilung nach der Einrichtung der Unterdruckkammer. Undertryckskammare $=$ Unterdruckkammer.

kam man zum Narkosezimmer durch einen viereckigen Durchgang, der auch eine Tür zum Korridor zwischen Vorhalle und Narkosezimmer hatte. Diesen Durchgang habe ich für meine Zwecke benutzt. Er ist vollständig von dem Verbandszimmer durch eine neuerrichtete Mauer, die aus Eisenbalken, Stahlnetz, Gips und Mörtel hergestellt ist, getrennt. Die Tür zum Korridor ist zugemauert. In der Wand zum Narkosenraum ist die oben 
beschriebene Tür angebracht. Eine neue Türöffnung hat man zwischen Verbandzimmer und Waschzimmer, in das alle Sterilisierungsapparate eingestellt worden, herstellen müssen.

Die auf diese Weise gewonnene Unterdruckkammer hat eine Größe von $2,34 \times 2,97 \mathrm{~m}$ und eine Höhe von $3,7 \mathrm{I} \mathrm{m}$. Um alle elektrischen Leitungen, die in die Mauer eingemauert sind, und um alle Stromunterbrecher hat man mit Gips oder Kitt sehr gewissenhaft dichten müssen, weil hier die Luft sonst durchdringt.

Die Luftverdünnung erhält man durch einen Ventilator, der im Keller aufgestellt ist. Die Lufttrommel, die zu diesem geht, hat einen Durchmesser von $12 \mathrm{~cm}$ und über sich in der Kammer eine perforierte vernickelte Kappe, die einige Zentimeter über dem Boden hervorsteht, Letztere steht in einer Ecke.

Der Motor, von 1/2 PS., entwickelt eine Geschwindigkeit von 1400 Umdrehungen in der Minute. Die Kraft des Motors wird auf den Ventilator durch Riemen übertragen. Der Ventilator macht 3800 Umdrehungen in der Minute. Bewegt sich der Ventilator mit maximaler Geschwindigkeit, so verursacht er ein ziemlich lautes Geräusch, aber doch nicht so laut, daß es die Operation stört. Beim gewöhnlichen Gange (bei einer Druckdifferenz von 5-10 cm Wasser) hört man ihn kaum.

An der Tür ist ferner eine $100 \times 32 \mathrm{~cm}$ große Eisenplatte befestigt, auf welcher alle erforderlichen Apparate montiert sind. Dadurch, daß alles auf einer Platte zu sitzen kommt, sind mit einem Schlage alle Schwierigkeiten betreffs der Lecke überwunden. Alle auf der Platte befestigten Apparate werden nämlich von dem Mechaniker luftdicht montiert. Die große Platte ist dann an der Tür nur festzuschrauben. Die Löcher, welche in der 'Tür behufs Durchleitung von Rohren angebracht werden müssen, sind dann hinsichtlich des Leckens ohne Bedeutung.

Ganz unten an der Platte (Fig. I und 2) sitzt ein Rheostat (A), durch welche die Geschwindigkeit des Motors und des Ventilators und auf diese Weise mit Leichtigkeit auch die Aussaugung reguliert wird. Links an der Platte sitzt ein Wassermanometer (C), das in Zentimetern die Druckdifferenz in und außerhalb der Kammer angibt; rechts findet sich ein Zuleitungsrohr (B) für Luft von außen. Durch zwei gegeneinander verschiebbare perforierte Scheiben kann die Luftzufuhr reguliert bzw. ganz ab- 
gestellt werden. Die innere Mündung ist gegen die Decke gerichtet, damit die einströmende Luft weder den Patienten noch den Operateur treffen soll. Dieses Rohr verschafft der Kammer nach Bedarf frische Luft und trägt zur Regulierung des Luftdruckes bei. In der Mitte der Platte ist der zur Maske gehende Aluminiumschlauch $(\mathrm{E})$, der die atmosphärische Luft in die Lungen des Patienten führt, sowie auch der Narkoseapparat $(G)$ montiert. Mittelst eines Dreiwegehahns sind 3 Einstellungen möglich: I. vollständige Absperrung der Luft, 2. nur Luftzufuhr, 3. Luftnarkosengasmischung.

Der Narkosenbehälter, in $\mathrm{mm}^{3}$ gradiert, wird in der Kammer nach Abschrauben eines Deckels von oben gefüllt.

Der Aluminiumschlauch ist biegsam, hat eine Länge von $100 \mathrm{~cm}$ und einen Durchmesser von $6 \mathrm{~cm}$. Irgendwelche Schwierigkeit oder Lästigkeit durch ein so langes Rohr zu atmen, besteht nicht.

Die Maske (D) ist eine gewöhnliche, über Nase und Mund legbare Maske. Die luftdichte Verbindung mit der Haut wird durch das elastische Gummi, welches gegen die Haut angedrückt wird, bewirkt. Der zur Maske gehörige Gummiteil kann abgenommen und gekocht werden. Auch der lange Schlauch kann nach Bedarf gekocht werden.

Der Hahn hätte leicht so konstruiert werden können, daß ich mehrere Kombinationsmöglichkeiten, z. B. Luft und Sauerstoff, nur Sauerstoff, Sauerstoff und Narkosengase, dadurch hätte zustande bringen können. Da dies doch ganz unnötig den Apparat kompliziert und verteuert hätte, habe ich davon Abstand genommen.

Sauerstoff - ein Sauerstoffzylinder (I) mit Reduzierungsventil - steht immer in der Kammer zur Hand. Vom Zylinder läuft ein biegsames Rohr $(\mathrm{K})$ direkt zur Maske. Während einer mit Druckdifferenz vorgenommenen Operation wird der Sauerstoff in die Lungen mit der Luft eingesaugt. An der Maske findet sich ferner, falls nötig, ein Ventil $(\mathrm{H})$, das durch gegeneinander verschiebbare perforierte Scheiben geöffnet und geschlossen werden kann.

Die Kammer, deren Wände und Decke sehr genau gekittet und mit weißer, harter Ripolinfarbe gestrichen sind, ist mit 
elektrischer Beleuchtung versehen. Der Sicherheit wegen geschieht die Beleuchtung von zwei voneinander unabhängigen Stromsystemen her. In der Kammer findet sich auch ein elektrischer Kochapparat. Das Zimmer ist so geräumig, daß Operateur, zwei Assistenten, drei Pflegerinnen ohne Schwierigkeit dort sich aufhalten können. In der Kammer steht auch ein besonderer Schrank zur Aufbewahrung von Medikamenten sowie von allen bei einer Lungen- und Herzoperation in Frage kommenden Instrumenten, welche im Bedarfsfalle während der Operation herausgenommen werden können. Während der Operation kann nichts von außen geholt werden.

Da diese Kammer dicht neben dem Operationssaal liegt, ist eine Überführung des Operationstisches nebst Patienten, falls während einer im gewöhnlichen Operationssaal angefangenen Operation eine Fortsetzung derselben mit Druckdifferenz sich als notwendig herausstellen sollte, leicht zu bewerkstelligen. Der ganze Apparat ist auch seitens eines ganz ungewohnten Assistenten leicht zu hantieren.

Die Tür wird geschlossen, der Kontakt (L) wird in die Wand gesetzt, wonach mit dem Rheostat die Aussaugung auf die gewünschte Höhe gebracht wird. In einigen Sekunden sinkt der Druck der Kammer, falls gewünscht, um I $5 \mathrm{~cm}$ Wasser. Mittelst Rheostaten und des Zufuhrventils ist der Druck zwischen o- $15 \mathrm{~cm}$ Wasser fast augenblicklich zu variieren. Luft- und Narkosengaszufuhr zur Maske wird durch den Griff (F) reguliert. Braucht man Sauerstoff, so ist nur das Sperrventil des Zylinders zu öffnen. Ehe die Luft der Kammer verdünnt wird, wird die Maske an das Gesicht des Patienten, wo sie während der Operation dann liegen bleibt, gedrïckt.

Meine Kammer hat den Vorteil gegenüber anderen Unterkammern, daß der Operateur und Narkotiseur in demselben Zimmer in vollständiger Verbindung miteinander stehen. Weiter hat der Operateur nebst den Assistenten volle „Ellenbogenfreiheit" und operiert unbehindert. Der Patient ist auch nicht mit dem Hals an der Wand fixiert, was auch bei verschiebbarem Halsring die freie Beweglichkeit des Patienten unmöglich macht und den Operateur bei Operationen in Brustapertur und im oberen Thoraxgebiet behindert. 
I84 Giertz, Eine neue Modifikation der Sauerbruchschen Unterdruckkammer.

Wie alle Maskenapparate hat auch mein Apparat die Nachteile, die ein eventuelles Erbrechen mit sich bringt, und ihm haftet die Gefahr an, die eine eventuelle akute Dilatation des Magens herbeiführen kann.

Meine Kammer hat den großen Vorteil, nicht zu viel weder bei der Anschaffung noch im Betriebe - zu kosten. Der Motor und der Ventilator sind nicht allzu teuer. Die Platte mit Schlauch, Maske und sonstigen Anordnungen ist für einen mäßigen Preis bei Stille-Stockholm zu haben. Auch der Betrieb ist billig. Schließlich hat die Kammer den Vorteil, als Untersuchungs- und Operationsdunkelzimmer für Endoskopie angewandt werden zu können, wenn sie als Unterdruckkammer nicht benutzt wird. Bei den Operationen, die ich in derselben ausgeführt habe, hat sie auf allerbeste Weise funktioniert. Sie ist jetzt acht Monate im Gebrauch gewesen, ohne jemals zu versagen. 